\title{
Dynamin-related protein 1 deficiency impairs mitophagy and accelerates lipopolysaccharide- induced inflammation in mice
}

Lixiang Wang ( $D$ ourika0211@yahoo.co.jp)

Kurume University School of Medicine https://orcid.org/0000-0003-2087-4787

Xin Li

New York University College of Dentistry

Yuki Hanada

Osaka University https://orcid.org/0000-0003-4610-9338

Nao Hasuzawa

Kurume University School of Medicine

Masatoshi Nomura

Kurume University School of Medicine

Ken Yamamoto

Kurume University School of Medicine https://orcid.org/0000-0002-1531-9685

\section{Article}

Keywords: lipopolysaccharide, Dynamin-related protein 1 (DRP1)

Posted Date: October 8th, 2020

DOl: https://doi.org/10.21203/rs.3.rs-82796/v1

License: (9) (i) This work is licensed under a Creative Commons Attribution 4.0 International License. Read Full License

Version of Record: A version of this preprint was published at Communications Biology on July $21 \mathrm{st}$, 2021. See the published version at https://doi.org/10.1038/s42003-021-02413-6. 


\section{Abstract}

Mitochondrial fusion and fission, which are strongly related to normal mitochondrial function, are referred to as mitochondrial dynamics. Mitochondrial fusion defects in the liver cause a non-alcoholic steatohepatitis-like phenotype and liver cancer. However, whether mitochondrial fission defect directly impair liver function and stimulate liver disease progression, too, is unclear. Dynamin-related protein 1 (DRP1) is a key factor controlling mitochondrial fission. We hypothesized that DRP1 defects are a causal factor directly involved in liver disease development and stimulate liver disease progression. We administered lipopolysaccharide (LPS) to liver-specific Drp1-knockout (Drp1LiKO) mice. We observed an enhanced inflammatory response accompanied by mitophagy impairment. Drp1 defects directly promoted hepatocyte apoptosis and subsequently induced infiltration of inflammatory macrophages enhanced inflammasome activation in the liver and increased pro-inflammatory cytokine expression in the liver and serum. Drp1 deletion increased the expression of numerous genes involved in the immune response and DNA damage in Drp1LiKO mouse primary hepatocytes. This is a novel mechanism of liver disease development in which Drp1 defect-induced mitochondrial dynamics dysfunction directly regulates the fate and function of hepatocytes and enhances LPS-induced acute liver injure in vivo.

\section{Introduction}

Mitochondria play a critical role in maintaining hepatocyte integrity and function, and mitochondrial dysfunction leads to liver diseases ${ }^{12} 34$. Mitochondrial function and morphology are interdependent, and the latter is shaped by ongoing mitochondrial fusion and fission (mitochondrial dynamics) ${ }^{567}$. In vertebrates, mitofusion-1/2 (MFN1/2) and optic atrophy 1 (OPA1) control mitochondrial fusion, while dynamin-related protein 1 (DRP1) and its receptors control mitochondrial fission. Mitochondrial dynamics research in recent years has shown the functional importance of mitochondrial dynamics in liver diseases. Kim et al. $(2013,2014)$ reported the impact of viral infection on mitochondrial dynamics, and mitochondrial dynamics alterations are used by hepatitis $B$ virus ${ }^{8}$ and hepatitis $C$ virus ${ }^{9}$ for maintenance of persistent infection. Overall, mitochondrial dynamics disruption promotes viral pathogenesis. Mitochondrial dynamics and liver diseases are at a crossroads. Studies have reported abnormal mitochondrial dynamics in other liver pathophysiological conditions, too. Cadmium is a long-lived environmental and occupational pollutant, and cadmium hepatotoxicity induces DRP1-dependent mitochondrial fragmentation by disturbing calcium homeostasis ${ }^{10}$. Mitochondrial dynamics are also related to the mechanism underlying acetaminophen-induced acute liver damage. Acetaminophen changes mitochondrial DRP1 levels, and when the DRP1 inhibitor Mdivi-1 inhibits mitochondria fission, acetaminophen induces greater hepatic impairment ${ }^{11}$. Alcoholic animal models also show mitochondrial morphology alteration. Mitochondria in normal hepatocytes usually show relatively slow dynamics, which are sensitive to inhibition by ethanol exposure ${ }^{12}$. MFN2 ablation in the liver causes endoplasmic reticulum (ER)-mitochondrial phosphatidylserine transfer defects, leading to a non-alcoholic steatohepatitis (NASH)-like phenotype and liver cancer ${ }^{13}$. 
We have reported that a mitochondrial fission defect in liver-specific Drp7-knockout (Drp1LiKO) mice, which demonstrate ER stress-promoted fibroblast growth factor 21 expression, subsequently functions as a metabolic regulator with anti-obesity and anti-diabetes effects. In addition, hematoxylin and eosin (H\&E) staining of Drp1LiKO mouse liver sections shows a disorganized lobular parenchyma with inflammatory cell infiltration ${ }^{14}$. Our previous studies have also reported that Drp1 disruption in the liver changes the expression of genes involved in the immune system in the liver. Gene Ontology (GO) biological studies have reported that 7 of the top 10 clusters are related to the immune system. These clusters include terms such as "immune response," "phagocytosis," "antigen processing," and "presentation" 15 .

In this study, we hypothesized that DRP1 deficiency-induced mitochondrial fission defects directly lead to liver disease development. In addition, we hypothesized that Drp1 defects stimulate liver disease progression. The aim was to determine the relationship between mitochondrial fission defects and liver disease progression. We tested our hypotheses in an acute liver injury experimental mouse model via lipopolysaccharide (LPS)-induced endotoxin shock. This is a novel mechanism involved in DRP1 defectinduced liver inflammation progression, which will provide insight into the role of DRP1 in liver function and acute liver injury.

\section{Results}

\section{Increased inflammatory response in LPS-treated Drp1 LiKO mice}

LPS administration increased messenger RNA (mRNA) levels of tumor necrosis factor alpha (Tnfa), interleukin 6 (I/6), interleukin 1 beta (I/1b), interferon beta 1 (Ifnb1), monocyte chemoattractant protein-1 (Mcp 1), and the NLR family pyrin domain containing 3 (N/rp3), which peaked at $1 \mathrm{~h}$ in the liver (Supplementary Fig. 1a). LPS-induced liver TNF, IL-6, interleukin 10 (IL-10) and MCP1 levels peaked at $1 \mathrm{~h}$, IL-1 $\beta$ levels peaked at $4 \mathrm{~h}$, and interferon gamma (IFN- $\gamma$ ) levels peaked at $8 \mathrm{~h}$ (Supplementary Fig. $1 \mathrm{~b}$ ). LPS-induced serum TNF and IL-10 levels peaked at $1 \mathrm{~h}$; MCP1 level peaked at $4 \mathrm{~h}$; and IL- 6 and IFN- $\gamma$ levels peaked at $8 \mathrm{~h}$ (Supplementary Fig. 1c).

Next, we compared the responses to LPS in control and Drp1LiKO mice after LPS injection. Drp1LiKO mice developed more severe symptoms of endotoxin shock, such as lack of activity and hunched back posture. Consistent with this observation, we also observed a significant increase in serum TNF levels at $1 \mathrm{~h}$ and IL-6, IL-1 3, MCP1, and IFN- $\gamma$ levels at $8 \mathrm{~h}$ after LPS injection in Drp1LiKO mice compared to control mice (Fig. 1a). In addition, liver Tnfa, II6, I/1b, Ifnb1, and Mcp1 mRNA expression levels significantly increased in LPS-treated Drp1LiKO mice compared to control mice. An interesting finding was a decrease in Drp1 mRNA levels in control mice, probably through transcriptional inhibition (Fig. 1b). We also detected a significant increase in liver TNF, IL-10, IL-1 $\beta$, MCP1, and IFN- $\gamma$ levels in LPS-treated Drp1LiKO mice (Supplementary Fig. 2a). 
To evaluate the degree of functional damage in the liver, we further evaluated the serum levels of alanine aminotransferase (ALT) and aspartate transaminase (AST), which are two markers of hepatocellular injure or necrosis. Both alanine aminotransferase (ALT) and aspartate transaminase (AST) significantly increased in LPS-treated Drp1LiKO mice (Fig. 1C), indicating that a high LPS concentration induces hepatocyte death in the absence of DRP1. Western blot analysis showed that activation of NLRP3 inflammasome pathways during the LPS-induced inflammatory response in the liver, and NLRP3 inflammasome marker, IL-1 $\beta$, and NLRP3 levels significantly increased in LPS-treated Drp1LiKO mice compared to control mice (Figs. 1d and 1e). Western blot analysis of the liver protein samples showed that LPS induces an inflammatory response via nuclear factor kappa B (NF-KB) and mitogen-activated protein kinase (MAPK) pathways, in addition to the NLRP3 inflammasome pathway, while there was no difference in NF-KB and MAPK pathways between control and Drp1LiKO mice (Supplementary Figs. 2b and 2c). In addition, phosphorylation of eukaryotic translation factor $2 a$ (P-elF2a) levels significantly increased in LPS-treated Drp1LiKO mice, indicating an increase in ER stress in Drp1LiKO mice (Supplementary Figs. $2 \mathrm{~b}$ and $2 \mathrm{c}$ ), which is consistent with our previous study which reported that DRP1 deletion in the liver causes ER stress via the elF2a pathway ${ }^{14}$.

\section{Increased apoptosis and inflammasome overactivation in LPS-treated Drp1 LiKO mice}

Macrophages represent a key cellular component of the liver, and are essential for maintaining tissue homeostasis and ensuring rapid response to hepatic injure ${ }^{16} 17$. Considering the observation on enhanced inflammasome pathway in Drp1LiKO mice, we went on to investigate the inflammasome activation in macrophages. To induce a maximum release of IL-1 $\beta$, these mice were intraperitoneal injected (IP) with $10 \mathrm{mg} / \mathrm{kg}$ body weight LPS for 4 hours and received an additional $50 \mu \mathrm{l}$ of $100 \mathrm{mM}$ ATP 30 minutes before sacrifice. H\&E staining confirmed inflammatory cell infiltration in both saline- and LPS/adenosine triphosphate (ATP)-treated livers of Drp1LiKO mice (Fig. 2a). Terminal deoxynucleotidyl transferase dUTP nick end labeling (TUNEL) staining showed that apoptotic hepatocytes significantly increased in saline-treated (Ctrl $8.67 \pm 1.86$ vs. KO $47.33 \pm 6.17$ ) and LPS/ATP-treated (Ctrl $42.00 \pm 6.08$ vs. KO $113.33 \pm 10.87$ ) livers of Drp1LiKO mice (Figs. 2a and 2b). Consistent with this observation, serum ALT and AST levels also significantly increased in LPS/ATP-treated Drp1LiKO mice (Supplementary Fig. 3a). Consistent with our previous observations (Fig. 1b), liver Tnfa, I/6, I/1b, F4/80, Mcp1, and NIrp3 mRNA expression significantly increased in Drp1LiKO mice compared to control mice after LPS/ATP treatment (Supplementary Fig. 3b).

Macrophages, neutrophils, T cells and B cells are cell types known for their role in the process of liver inflammation, to determine the composition of inflammatory cell clusters in Drp1LiKO mice especially which cell types are predominantly expressed in these cell clusters, we performed immunostaining in liver tissues with various immune markers including adhesion $G$ protein-coupled receptor E1 (F4/80) for macrophages, lymphocyte antigen 6 complex, locus $\mathrm{G}(\mathrm{Ly} 6 \mathrm{G})$ for neutrophils, CD3 antigen, epsilon polypeptide (CD3) for T cells and protein tyrosine phosphatase, receptor type, C (B220) for B cells (Fig. 2a and Supplementary Fig. 4a). Cell-counting results are summarized in Fig. 2c and Supplementary Figs. $4 b-d$. We found no differences in the number of T-cells (Supplementary Fig. 4b) and neutrophils 
(Supplementary Fig. 4d), but the number of B-cells increased (saline Ctrl $183.33 \pm 26.64$ vs. saline KO $367.00 \pm 22.50$; LPS/ATP Ctrl $206.33 \pm 39.30$ vs. LPS/ATP KO $382.33 \pm 29.85$ ) (Supplementary Fig. 4c), and F4/80-positive macrophages were the largest population of the increased inflammatory cells (saline Ctrl $2401.50 \pm 243.40$ vs. saline KO $6097.75 \pm 1089.91$; LPS/ATP Ctrl $4851.25 \pm 167.44$ vs. LPS/ATP KO $8630.50 \pm 904.51$ ) (Fig. 2c). Interestingly, immunostaining analysis also revealed co-immunostaining of IL-1 $\beta$ with F4/80-positive macrophages, indicating specific co-localization of IL-1 $\beta$-positive cells with macrophages, (Fig. $2 d$ and Supplementary Fig. 4 e), that is, high IL-1 $\beta$ expression in macrophages. We also observed a clear line of liver-resident Kupffer cells along the sinusoid wall, which constituted the main macrophage population in the livers of control mice (Fig. 2d, Zoom1). In contrast, macrophages in Drp1LiKO mice showed an abnormal morphology with irregular location and distribution patterns. In addition to main macrophages, we found two other distinct macrophage populations, either derived from infiltrating monocytes or derived from activated Kupffer cells. The majority of these macrophages showed low F4/80 expression but significantly high IL-1 $\beta$ expression after LPS/ATP treatment (Fig. 2d, Zoom2 and Zoom3), and only a few macrophages showed high F4/80 expression but no IL-1 $\beta$ expression after LPS/ATP treatment (Fig. 2d, Zoom4).

\section{Inflammatory M1 macrophage infiltration in Drp1 LiKO mice}

It is possible that different populations of hepatic macrophages in control and Drp1LiKO mice exert distinct functions and contribute to differences in the response to an acute LPS challenge. During acute inflammation, M1-polarized macrophages express high IL-1 $\beta$ levels, while IL-1 $\beta$ levels are undetectable in M2-polarized macrophages ${ }^{18}$. To further explore the polarization signature of these macrophages in the liver of control and Drp1LiKO mice, we performed flow cytometry assay and sequential gating analysis using isolated non-parenchymal liver cells without LPS injection. M1 and M2 macrophages were identified as $C D 45^{+} \mathrm{CD} 11 \mathrm{~b}^{+} \mathrm{F} 4 / 80^{+} \mathrm{Ly} 6 \mathrm{C}^{\text {High }}$ and $7 \mathrm{AAD}{ }^{-} \mathrm{CD} 45^{+} \mathrm{CD} 11 \mathrm{~b}^{+} \mathrm{F} 4 / 80^{+} \mathrm{CD} 206^{\text {High }}$ macrophages, respectively (Figs. 3a and $3 b$ ). Sequential gating analysis showed a significant increase in Ly $6 \mathrm{C}^{\text {High }} \mathrm{M} 1$ macrophages (Ctrl $24.96 \% \pm 1.16 \%$ vs. KO $44.10 \% \pm 3.77 \%$ ) and a significant decrease in the CD206 High M2 macrophages in Drp1LiKO mice (Ctrl $49.16 \% \pm 1.07 \%$ vs. KO $37.42 \% \pm 2.53 \%$ ) (Fig. 3c). IL-1 $\beta$ with F4/80 co-immunostaining confirmed that liver-resident Kupffer cells, which lined the sinusoid wall in control mice and macrophages in Drp 1LiKO mice, have different location and distribution patterns. Some macrophages with low F4/80 expression showed IL-1 $\beta$ production even without any stimulation in Drp1LiKO mice but not in control mice (Fig. 3d). Therefore, Drp1 loss in hepatocytes induces macrophage activation, and most of these activated macrophages are prone to differentiating into class M1 macrophages.

\section{Decreased autophagy formation in LPS-treated Drp1 LiKO mice}

Autophagy is reported to be involved in the mitochondrial dynamics and mitochondrial quality control, furthermore, it is reported that autophagosomes form at the mitochondria- ER contact site (MAM) in mammalian cells ${ }^{19}$. In our previous study, we have demonstrated that loss of DRP1 leads to MAM defect, therefore, we investigated whether DRP1 defect alters autophagy formation in LPS treated hepatocytes. 
The autophagy pathways in the LPS-induced inflammatory response were activated, and the autophagy activation marker light chain 3-phospholipid-conjugated (LC3-II) expression significantly increased and peaked at $8 \mathrm{~h}$ after LPS treatment in control mice, while LC3-II expression significantly decreased in Drp1LiKO mice compared to control mice (Figs. 4a and 4b). It is known that the degradation of sequestosome1 (SQSTM1/P62) by the autophagic-lysosome pathway and the deficient autophagy could lead to P62 accumulation at protein levels ${ }^{20} 21$. In addition, western blot analysis showed a significant increase in P62 accumulation in Drp1LiKO mice (Figs. 4a and 4c). Mitophagy is the selective pathway degradation of mitochondria by autophagy. phosphatase and tensin homolog-induced putative kinase 1 (PINK1) leads to mitophagy of damaged mitochondria 222324 . In healthy mitochondria, which have a well-maintained mitochondrial membrane potential $\left(\Delta \Psi_{m}\right)$, PINK1 levels remain low or undetectable. An acute LPS challenge led to PINK1 accumulation in both control and Drp1LiKO mice. There was a greater increase in PINK1 levels in Drp 1LiKO mice at 1 and $8 \mathrm{~h}$, which was sustained to $24 \mathrm{~h}$ after LPS treatment (Figs. 4a and 4d), indicating that LPS treatment induces mitophagy impairment and that mitophagy impairment is further disturbed by Drp1 defects in Drp1LiKO mice.

Immunofluorescence microscopy showed a significant decrease in LC3-positive dots in Drp 1LiKO mice compared to control mice after LPS/ATP treatment (Fig. 4e). Of note, localization of LC3-positive cells was different from that of IL-1 $\beta$-positive macrophages, indicating that autophagy occurs in hepatocytes but not in macrophages. Western blot analysis also confirmed that the LC3-II in hepatocytes significantly decreased in Drp1LiKO mice compared to control mice after LPS/ATP treatment (Fig. 4f).

\section{Increased cell death, ER stress, inflammatory response and decreased $\Delta \Psi_{\mathrm{m}}$ in Drp1LiKO mouse primary hepatocytes}

So far, our results showed that DRP1 disruption in the liver can lead to mitophagy defects and hepatocyte apoptosis, which might trigger enhanced infiltration and polarization of macrophages. To gain further insight into this mechanism, we carried out further experiments with primary hepatocytes from control and Drp 1 knockout mice. Isolation and culture of the hepatocytes were performed using a two-step collagenase perfusion method as described previously ${ }^{25}$. When examined and counted under a light microscope using a hemocytometer chamber, the cell yield and viability were less in Drp1LiKO mice $(11.43 \% \pm 1.38 \%)$ compared to control mice $(100.00 \% \pm 12.20 \%)$, indicating that DRP1 disruption in the liver impairs hepatocyte survival (Fig. 5a).

ER stress evokes upregulation of the nuclear protein 1 (Nupr1; p8), Tribbles homolog 3 (Trib3), and DNA damage-inducible transcript 3 (Ddit3; Chop) in Drp1 LiKO mice ${ }^{14}$, and these genes are related to ER stress and ER stress-mediated cell apoptosis 262728 . Real-time polymerase chain reaction (PCR) analysis confirmed the mRNA expression of ER stress genes significantly increased in Drp1LiKO mouse primary hepatocytes (Fig. 5b). Next, to fully reveal the gene expression altered by Drp1 deletion, we conducted an independent microarray analysis with primary hepatocyte harvested 24 hours after seeding. Genes were selected using the criterion of a $Z$ score of $\geq 2$, which identified 680 up-regulated and 559 down-regulated genes in Drp1LiKO cells. We use the DAVID tool to functionally cluster up-regulated and down-regulated 
genes by similarly annotated gene ontology (G0) biological process terms, respectively. The top 5 significantly enriched annotation clusters of upregulated genes are shown in Fig. $5 \mathrm{c}$. All 5 clusters were related to the immune system: the immune system process, defense response to virus, innate immune response, response to virus, and immune response. Genes involved in the immune system process are shown in Supplementary Table 4. The most significant 5 clusters for downregulated genes were related to mitotic nuclear division, cell cycle, cell division, chromosome segregation, and mitotic chromosome condensation. Genes involved in mitotic nuclear division are shown in Supplementary Table 5. These data are consistent with previous studies that reported that DRP1 loss leads to cell arrest, replication stress, centrosome overduplication, and chromosomal instability, subsequently increasing DNA damage and cell apoptosis ${ }^{29}$. Overall, these findings showed that Drp1 deletion increases the expression of numerous genes involved in the immune response and induces DNA damage in primary hepatocytes.

We further compared the mitochondrial morphology using Mito Tracker Red, a specific mitochondria fluorescence probe. In control mouse primary hepatocytes (Fig. 5 d), $57.17 \% \pm 4.00 \%$ of the mitochondria were fragmented, with only $14.80 \% \pm 2.35 \%$ hepatocytes showing tubular morphology. Drp1LiKO mouse primary hepatocytes containing fragmented mitochondria decreased to $10.50 \% \pm 2.66 \%$, while those containing tubular mitochondria increased to $65.70 \% \pm 3.77 \%$. These results indicated less mitochondrial fission and more mitochondrial fusion in Drp1LiKO mouse primary hepatocytes compared to control mouse primary hepatocytes.

The mitochondrial respiratory chain generates a membrane potential across the mitochondrial inner membrane as protons are pumped across the inner membrane. $\Delta \Psi_{\mathrm{m}}$ regulates matrix configuration and cytochrome $c$ release during apoptosis ${ }^{30}$. As shown in Fig. $5 e$, a greater percentage of hepatocytes with low $\Delta \Psi_{\mathrm{m}}$ were found in the Drp $1 \mathrm{LiKO}$ hepatocyte culture compared to the control mouse primary hepatocyte culture (Ctrl $17.68 \% \pm 1.45 \%$ vs. KO $42.46 \% \pm 3.88 \%$ ).

\section{Decreased mitophagy formation and increased inflammatory response in LPS-treated Drp1 LiKO mouse primary hepatocytes}

OPA1 is a mitochondrial fusion protein that resides in the inner mitochondrial membrane, and OPA1 and LC3 co-localization indicates the sequestration of mitochondria inside autophagosomes (Fig. 6a). We observed that $5.07 \% \pm 0.50 \%$ of mitochondria labeled with optic atrophy 1 (OPA1) co-localized with LC3 punctate foci in control mouse primary hepatocytes, indicating that mitophagy occurs in $5.07 \% \pm 0.50 \%$ of the total mitochondria in control mouse primary hepatocytes after LPS treatment. In contrast, mitophagy decreased to $0.22 \% \pm 0.10 \%$ of the total mitochondria in Drp 1 LiKO mouse primary hepatocytes (Fig. 6 b). In addition, $49.43 \% \pm 1.96 \%$ of positive LC3 signals were also positive for OPA 1 in control mouse primary hepatocytes, indicating that around half of the total autophagy is mitophagy. The percentage of mitophagy in total autophagy significantly decreased to $6.92 \% \pm 2.23 \%$ in Drp 1 LiKO mouse primary hepatocytes (Fig. 6c). 
We also detected significantly increased TNF, IL-6, and IL-10 levels in Drp1LiKO hepatocyte culture supernatants (Fig. 6d). Consistent with these observations, we found a significant increase in Tnfa, $/ 16$, $\| / 1 b$, and Ifnb1 mRNA expression in Drp1LiKO mouse primary hepatocytes compared to control mouse primary hepatocytes (Fig. 6e).

\section{Discussion}

Many studies have reported the abnormal function of mitochondrial dynamics in liver disease. Although mitochondrial fusion defects in the liver cause a NASH-like phenotype and liver cancer, whether mitochondrial fission defects directly impair liver function and stimulate liver disease progression is unclear. In the present study, DRP1 defects lead to elongated mitochondria with low $\Delta \Psi_{m}$, DNA damage, and high expression of ER stress genes $P 8$, Chop, and Trib3, which, in turn, increase hepatocyte apoptosis in Drp1LiKO mice; the dead hepatocytes then release danger signals that lead to M1 inflammatory macrophage infiltration (Fig. 7). ER stress pathway protein-folding and quality control functions maintain cell homeostasis and are closely linked to mechanisms underlying immunity and inflammation 2631 . Potent and prolonged ER stress produces excess Trib3 and leads to apoptosis ${ }^{28}$. ER stress-inducible P8 is involved in several physiological and pathological processes ${ }^{32}$. CHOP promotes liver damage during acute liver failure by activating a key mediator to link ER stress and reactive oxygen species (ROS) ${ }^{33}$. Drp1LiKO mice evoke the ER stress pathway, in addition to DNA damage, to promote hepatocyte apoptosis and hepatitis. DRP1 deletion increases the expression of numerous genes involved in the immune response and DNA damage in Drp1LiKO mouse primary hepatocytes. A lack of fission by DRP1 downregulation leads to loss of mitochondrial DNA and a decrease in $\Delta \Psi_{m}$ in Hela cells ${ }^{29}$, since loss of $\Delta \Psi_{\mathrm{m}}$ is closely linked to cell apoptosis by various insults, we believe the increased population with low $\Delta \Psi_{\mathrm{m}}$ in Drp1LiKO mouse primary hepatocytes might be an insult and/or a consequence induced by increased cell apoptosis. Furthermore, DRP1 loss in the liver leads to an accelerated inflammatory response induced by LPS. Compared to control mice, Drp1LiKO mice show increased expression of proinflammatory cytokines in the liver and serum, increased infiltration of M1 inflammatory macrophages, enhanced inflammasome activation in liver, increased hepatocyte apoptosis, and decreased mitophagy formation in hepatocytes. Overall, these findings showed that upon an LPS challenge, mitophagy defects in hepatocytes and NLRP3 inflammasome overactivation in M1 inflammatory macrophages jointly contribute to the elevated inflammatory response in Drp1LiKO mice (Fig. 7). In conclusion, using acute liver injury experimental mouse model via LPS-induced endotoxin shock, our work provides a novel approach to study DRP1 defect-induced liver inflammation progression, which will provide new insight into the role of DRP1 in liver function and acute liver injure.

Mitochondrial dynamics and mitophagy have gained significant interest because these events modulate mitochondrial function during many physiological and pathological conditions. In a mouse embryonic fibroblast model, Drp1 mutant cells showed abnormal mitochondrial morphology and defective mitophagy, leading to the activation of sterile myocardial inflammation, resulting in heart failure ${ }^{34}$. In INS1 cells, inhibition of the fission machinery through a dominant-negative form of DRP1 (DRP1 ${ }^{\mathrm{K} 38 \mathrm{~A}}$ ) 
decreases mitochondrial autophagy and results in the accumulation of damaged mitochondrial material 35. In a transverse aortic constriction mouse model, DRP1-dependent mitochondrial autophagy plays a protective role against pressure overload-induced mitochondrial dysfunction and heart failure ${ }^{36}$. In addition, Parkin-independent mitophagy requires DRP1 and maintains the integrity of the mammalian heart and brain ${ }^{37}$. Insufficient mitophagy formation induced by DRP1 defects might increase the inflammatory response and enhance the severity of liver injury in response to multiple pathogens.

Hepatitis, refers to an inflammatory condition of the liver, is a serious global public health problem. Although it is usually caused by a viral infection, there are several other possible risk factors, including: infections, alcohol, toxins, drugs and autoimmune diseases. In this article, we demonstrated that mitochondrial fission defect caused by the lack of DRP1 lead to the tissue damage associated with hepatitis, suggesting that DRP1 could be a new therapeutic target for inflammatory liver diseases. Taken together, our study identifies mitochondrial fission defect directly impairs liver function and stimulates liver disease progression. Our study with Drp $1 \mathrm{LiKO}$ mice disclosed an essential role of mitochondrial fission in mitochondrial quality control for prevention of hepatic inflammation. This is an exciting discovery which offers a promising alternative approach to developing new therapeutic strategies for inflammatory liver disease.

\section{Methods}

\section{Animals and treatments}

We purchased 8-12-week-old male C57BL/6J mice from KBT Oriental Co., Ltd. (Saga, Japan). We created Drp1LiKO (Alb-Cre ${ }^{T g /+} \operatorname{Drp} 1^{\text {flox/flox }}$ ) and control (Drp $1^{\text {flox/flox }}$, sibling littermates) mice and genotyped them for the Drp1 conditional allele and the Cre transgene using PCR, as previously described 14 . The mice were maintained in a standard specific-pathogen free room at room temperature $\left(22^{\circ} \mathrm{C}-\right.$ $24{ }^{\circ} \mathrm{C}$ ) and $50 \%-60 \%$ relative humidity under a $12 \mathrm{~h} / 12 \mathrm{~h}$ light/dark cycle (lights off at 8:00 p.m.). The mice were fed a normal chow diet (NCD; 5.4\% fat, CRF-1; Orient Yeast, Tokyo, Japan) ad libitum. Experiments were performed between 10:00 and 11:00 a.m. after overnight fasting, except the LPS challenge and primary hepatocyte isolation (ad libitum).

All mouse procedures and protocols were approved by the Ethics Committees on Animal Experimentation (Kyushu University, Graduate School of Medicine, Japan) and performed in accordance with the Guide for the Care and Use of Laboratory Animals.

\section{Mouse Primary Hepatocyte Isolation And Culture}

We performed a rapid two-step method of isolating mouse primary hepatocytes, as previously described 25 . Briefly, we anesthetized the mice with pentobarbital sodium ( $50 \mathrm{mg} / \mathrm{kg}$ body weight) and perfused their liver tissue with prewarmed Hank's balanced salt solution supplemented with $0.5 \mathrm{mM}$ ethylene 
glycol tetraaceticacid (EGTA) and $10 \mathrm{mM}$ 4-(2-hydroxyethyl)-1-piperazineethanesulfonic acid (HEPES) for $5 \mathrm{~min}$, followed by digestion buffer $(1000 \mathrm{mg} / \mathrm{L}$ of low-glucose Dulbecco's modified Eagle's medium [DMEM] supplemented with $0.8 \mathrm{mg} / \mathrm{mL}$ of collagenase type 1 ; Worthington Biochemical Corporation, Lakewood, NJ, USA). Next, we filtered single-cell suspensions through a $70 \mu \mathrm{m}$ cell strainer (BD Falcon, Bedford, MA, USA) and centrifuged them at $50 \times g$ for $1 \mathrm{~min}$ and collected the cell supernatants separately for the preparation of nonparenchymal cells, as described later. We washed the hepatocytes in the pellet twice, suspended them in $4500 \mathrm{mg} / \mathrm{L}$ of high-glucose DMEM supplemented with $1 \mu \mathrm{M}$ insulin, $2 \mathrm{mM}$ L-glutamine, $10 \mathrm{IU} / \mathrm{mL}$ of penicillin, $10 \mathrm{IU} / \mathrm{mL}$ of streptomycin, and $10 \%$ fetal bovine serum, and seeded them on 6-well plastic plates coated with collagen type I (Cellmatrix type I-P, Nitta Gelatin, Osaka, Japan) at a density of 300,000 cells/well. After $4 \mathrm{~h}$ incubation in a $5 \% \mathrm{CO}_{2}$ incubator at $37^{\circ} \mathrm{C}$, we changed the culture medium to discard floating unattached cells, and the growth medium was changed daily.

\section{Mouse Hepatic Nonparenchymal Cell Isolation And Flow Cytometry}

To further explore the polarization signature of macrophages in the liver of control and Drp1LiKO mice, we performed flow cytometry assay and sequential gating analysis using isolated nonparenchymal hepatocytes without LPS injection. We prepared hepatic nonparenchymal cell containing suspensions from the liver, as described before. The suspensions were centrifuged at $50 \times g$ for $5 \mathrm{~min}$ to remove the remaining hepatocytes, and the resulting cell suspensions were pelleted by centrifugation at $800 \times g$ for 5 min and resuspended in phosphate-buffered saline (PBS) supplemented with $5 \%$ bovine serum albumin. Next, we stained cells with appropriate antibodies (Supporting Table 1) for 30 min on ice, washed them, and analyzed them using a NovoCyte flow cytometer (ACEA Biosciences, San Diego, CA, USA) and NovoExpress software.

\section{Total RNA isolation, microarray procedures, and real-time- PCR}

We isolated total RNA from the mouse liver and primary hepatocytes using TRIzol Reagent (Invitrogen Corporation, Carlsbad, CA, USA) and purified it using the SV Total RNA Isolation System (Promega Corporation, Madison, WI, USA) according to the manufacturer's instructions. For microarray analysis, we amplified complementary RNA (cRNA) and labeled it using the Low input Quick Amp Labelling Kit (Agilent Technologies, Santa Clara, CA, USA). Next, we hybridized the cRNA to a 60K 60-mer oligomicroarray (SurePrint G3 Mouse Gene Expression Microarray $8 \times 60 \mathrm{~K}$ v2; Agilent Technologies) according to the manufacturer's instructions.

For real-time-PCR assays, we converted $500 \mathrm{ng}$ of total RNA into first-strand complementary DNA (cDNA) using the QuantiTect Reverse Transcription Kit (QIAGEN, Hilden, Germany) according to the manufacturer's instructions. Next, we used the cDNA for quantitative real-time PCR using the power $2 \times$ 
SYBR Green PCR Master Mix and monitored the process using an ABI Prism 7500 sequence detection system (Thermo Fisher Scientific, Rockford, IL, USA). Supporting Table 2 lists the primer sequences of the selected genes. We normalized relative gene expression versus control to Gapdh expression.

\section{Western blot analysis}

We homogenized fresh mouse liver tissue in western lysis buffer $(20 \mathrm{mM}$ Tris- $\mathrm{HCl}$ pH 7.6, $150 \mathrm{mM} \mathrm{NaCl}$, $2 \mathrm{mM}$ ethylenediaminetetraacetic acid [EDTA], 0.5\% NP40) containing protease and phosphatase inhibitor tablet (Roche Applied Science, Mannheim, Germany) and centrifuged it at 10,000 $\times g$ for 5 min at $4{ }^{\circ} \mathrm{C}$. We discarded the pellet and measured the protein content using a bicinchoninic acid (BCA) Protein Assay kit (Thermo Fisher Scientific). Next, the samples were mixed with Laemmli sample buffer (1:1; BioRad, Hercules, CA, USA) and heated for 5 min at $95^{\circ} \mathrm{C}$. We performed sodium dodecyl sulfate polyacrylamide gel electrophoresis (SDS-PAGE) on an equal amount of protein from each sample, transblotted it on a polyvinylidene difluoride (PVDF) membrane, and subjected it to immunoblot assay with primary antibodies, followed by horseradish peroxidase (HRP)-linked secondary antibodies. Supporting Table 1 lists the primary and secondary antibodies used. Finally, we visualized protein bands using the electrochemiluminescence (ECL) Western Blotting Detection System (GE Healthcare, Buckinghamshire, $\mathrm{UK})$.

\section{Histology, Immunohistochemistry, And Live-cell Imaging}

We fixed liver tissue and primary hepatocytes by immersion in $4 \%(\mathrm{w} / \mathrm{v})$ paraformaldehyde (PFA) overnight at $4{ }^{\circ} \mathrm{C}$, embedded the liver tissue in paraffin according to standard methods, and cut it into 5$\mu \mathrm{m}$-thick sections. For H\&E staining, we stained the paraffin sections with Mayer's hematoxylin solution for $5 \mathrm{~min}$, followed by counterstaining with $0.5 \%(\mathrm{v} / \mathrm{v})$ eosin alcohol solution for $2 \mathrm{~min}$. For immunostaining, we diluted primary and secondary antibodies in Can Get Signal ${ }^{\mathrm{TM}}$ Immunoreaction Enhancer Solutions A and B, respectively (Toyobo, Osaka, Japan). Supporting Table 1 lists the primary and secondary antibodies used. Next, we analyzed hepatocyte apoptosis using the TUNEL assay kit (Burlington, Boston, MA, USA) according to the standard protocol. The tissue sections were analyzed under a BZ-8000 microscope (Keyence, Osaka, Japan) or a confocal microscope LSM700 (Zeiss, Oberkochen, Germany).

For live-cell imaging, we seeded hepatocytes on a type l-coated $35 \mathrm{~mm}$ glass-based dish (Iwaki, Tokyo, Japan) preloaded with MitoTracker ${ }^{\circledR}$ Red, a specific mitochondria fluorescence probe. We further measured $\Delta \Psi_{m}$ using a fluorescence probe tetramethylrhodamine ethyl ester perchlorate (TMRE). Next, we examined the hepatocytes under the confocal microscope LSM700 and analyzed image data using ZEN software (Zeiss, Oberkochen, Germany). A hepatocyte was judged to have fragmented mitochondria if $<25 \%$ of the mitochondria visible had a length five times their width and tubular if $>75 \%$ of the mitochondria had a length five times their width. 


\section{Measurement of cytokine levels in the liver, serum, and cell culture supernatants}

LPS was injected intraperitoneally into mice at $5 \mathrm{mg} / \mathrm{kg}$ body weight, and liver and serum samples were collected from the inferior vena cava at different time points $(0,1,4,8,24$, and $48 \mathrm{~h})$. The liver tissue was perfused with cold PBS through the portal vein and then chopped into 1-2 mm pieces. Next, we added $100 \mathrm{mg}$ of liver tissue to $1 \mathrm{~mL}$ of cell lysis buffer (R\&D System, Minneapolis, MN, USA) and homogenized it using a tissue homogenizer. Finally, we diluted liver, serum, and cell culture supernatant samples with known high protein concentrations at 1:50 using an assay diluent and quantified the levels of various types of cytokines using the BD Cytometric Bead Array (CBA) Cytokine kit (Becton-Dickinson, Franklin Lakes, NJ, USA) and a NovoCyte flow cytometer (ACEA Biosciences, San Diego, CA, USA) and NovoExpress software.

\section{Biochemical assays}

To evaluate the degree of functional damage in the liver, we measured serum ALT and AST levels (two markers of hepatocellular injure or necrosis) using the DRI-CHEM3500 Chemistry Analyzer (Fujifilm, Tokyo, Japan).

\section{Statistical analysis}

All data were expressed as the mean \pm standard error of the mean (SEM). Two-tailed Student's $t$-test was performed to compare two groups using Microsoft Excel (Mac 2011 version 14.3.5; Microsoft Japan, Tokyo, Japan). Two-way analysis of variance (ANOVA) with Tukey's post hoc test or ordinary one-way ANOVA was performed to compare multiple groups using GraphPad Prism 6.0 software (GraphPad, San Diego, CA, USA). To detect an outlier, we performed Grubbs' test using a free statistical calculator (QuickCals, http://www.graphpad.com/quickcalcs/). Significance levels were set at $p<0.05, p<0.01$, and $p<0.001$.

\section{Declarations}

\section{Data Availability}

The data that support the findings of this study are available from the corresponding authors upon reasonable request. The microarray data from this publication have been submitted to the Gene Expression Omnibus database [http://www.ncbi.nlm.nih.gov/geo/] and assigned an identifier [accession: GSE156982].

\section{Author contributions}


LW, MN and KY designed the experiments. LW performed the experiments. $\mathrm{YH}$ and $\mathrm{NH}$ supported experiments. $\mathrm{LW}$ analyzed the data. $\mathrm{LW}$ and $\mathrm{XL}$ wrote the manuscript. $\mathrm{MN}$ and $\mathrm{KY}$ are the guarantors of the article.

\section{Acknowledgments}

We appreciate the technical assistance from The Research Support Center, Research Center for Human Disease modeling, Kyushu University Graduate School of Medical Sciences. This work was supported in part by the Japanese Society for the Promotion of Science (JSPS) KAKENHI (M. Nomura, Grant Number 23591356; L. Wang, Grant-in-Aid for Young Scientists B, Grant Number 16K19557).

\section{References}

1 Hassanein, T. \& Frederick, T. Mitochondrial dysfunction in liver disease and organ transplantation. Mitochondrion 4, 609-620, doi:10.1016/j.mito.2004.07.015 (2004).

2 Grattagliano, I. et al. Mitochondria in chronic liver disease. Curr Drug Targets 12, 879-893, doi:10.2174/138945011795528877 (2011).

3 Begriche, K., Massart, J., Robin, M. A., Bonnet, F. \& Fromenty, B. Mitochondrial adaptations and dysfunctions in nonalcoholic fatty liver disease. Hepatology 58, 1497-1507, doi:10.1002/hep.26226 (2013).

4 Koliaki, C. et al. Adaptation of hepatic mitochondrial function in humans with non-alcoholic fatty liver is lost in steatohepatitis. Cell Metab 21, 739-746, doi:10.1016/j.cmet.2015.04.004 (2015).

5 Detmer, S. A. \& Chan, D. C. Functions and dysfunctions of mitochondrial dynamics. Nat Rev Mol Cell Bio/ 8, 870-879, doi:10.1038/nrm2275 (2007).

6 Liesa, M. \& Shirihai, O. S. Mitochondrial dynamics in the regulation of nutrient utilization and energy expenditure. Cell Metab 17, 491-506, doi:10.1016/j.cmet.2013.03.002 (2013).

7 Wai, T. \& Langer, T. Mitochondrial Dynamics and Metabolic Regulation. Trends Endocrinol Metab 27, 105-117, doi:10.1016/j.tem.2015.12.001 (2016).

$8 \quad$ Kim, S. J. et al. Hepatitis B virus disrupts mitochondrial dynamics: induces fission and mitophagy to attenuate apoptosis. PLoS Pathog 9, e1003722, doi:10.1371/journal.ppat.1003722 (2013).

9 Kim, S. J. et al. Hepatitis C virus triggers mitochondrial fission and attenuates apoptosis to promote viral persistence. Proc Natl Acad Sci U S A 111, 6413-6418, doi:10.1073/pnas.1321114111 (2014). 
$10 \mathrm{Xu}, \mathrm{S}$. et al. Cadmium induced Drp1-dependent mitochondrial fragmentation by disturbing calcium homeostasis in its hepatotoxicity. Cell Death Dis 4, e540, doi:10.1038/cddis.2013.7 (2013).

11 Gao, Y. et al. Early Stage Functions of Mitochondrial Autophagy and Oxidative Stress in Acetaminophen-Induced Liver Injury. J Cell Biochem 118, 3130-3141, doi:10.1002/jcb.25788 (2017).

12 Das, S. et al. Mitochondrial morphology and dynamics in hepatocytes from normal and ethanolfed rats. Pflugers Arch 464, 101-109, doi:10.1007/s00424-012-1100-4 (2012).

13 Hernandez-Alvarez, M. I. et al. Deficient Endoplasmic Reticulum-Mitochondrial Phosphatidylserine Transfer Causes Liver Disease. Cell 177, 881-895 e817, doi:10.1016/j.cell.2019.04.010 (2019).

14 Wang, L. et al. Disruption of mitochondrial fission in the liver protects mice from diet-induced obesity and metabolic deterioration. Diabetologia 58, 2371-2380, doi:10.1007/s00125-015-3704-7 (2015).

15 Wang, L. \& Nomura, M. Loss of Drp1 in the liver leads to an alteration in expression of the genes involved in the immune system. Genom Data 6, 27-30, doi:10.1016/j.gdata.2015.07.021 (2015).

16 Pelegrin, P. \& Surprenant, A. Dynamics of macrophage polarization reveal new mechanism to inhibit IL-1 beta release through pyrophosphates. EMBO J 28, 2114-2127, doi:10.1038/emboj.2009.163 (2009).

17 Krenkel, O. \& Tacke, F. Liver macrophages in tissue homeostasis and disease. Nat Rev Immuno/ 17, 306-321, doi:10.1038/nri.2017.11 (2017).

18 Ju, C. \& Tacke, F. Hepatic macrophages in homeostasis and liver diseases: from pathogenesis to novel therapeutic strategies. Cell Mol Immuno/ 13, 316-327, doi:10.1038/cmi.2015.104 (2016).

19 Hamasaki, M. et al. Autophagosomes form at ER-mitochondria contact sites. Nature 495, 389-393, doi:10.1038/nature11910 (2013).

20 Watanabe, Y. \& Tanaka, M. p62/SQSTM1 in autophagic clearance of a non-ubiquitylated substrate. J Cell Sci 124, 2692-2701, doi:10.1242/jcs.081232 (2011).

21 Komatsu, M. \& Ichimura, Y. Physiological significance of selective degradation of p62 by autophagy. FEBS Lett 584, 1374-1378, doi:10.1016/j.febslet.2010.02.017 (2010).

22 Narendra, D. P. et al. PINK1 is selectively stabilized on impaired mitochondria to activate Parkin. PLoS Bio/ 8, e1000298, doi:10.1371/journal.pbio.1000298 (2010).

23 Geisler, S. et al. PINK1/Parkin-mediated mitophagy is dependent on VDAC1 and p62/SQSTM1. Nat Cell Biol 12, 119-131, doi:10.1038/ncb2012 (2010).

24 Vives-Bauza, C. et al. PINK1-dependent recruitment of Parkin to mitochondria in mitophagy. Proc Natl Acad Sci U S A 107, 378-383, doi:10.1073/pnas.0911187107 (2010). 
25 Severgnini, M. et al. A rapid two-step method for isolation of functional primary mouse hepatocytes: cell characterization and asialoglycoprotein receptor based assay development. Cytotechnology 64, 187-195, doi:10.1007/s10616-011-9407-0 (2012).

26 Sano, R. \& Reed, J. C. ER stress-induced cell death mechanisms. Biochim Biophys Acta 1833, 3460-3470, doi:10.1016/j.bbamcr.2013.06.028 (2013).

27 Cai, D. et al. Nupr1/Chop signal axis is involved in mitochondrion-related endothelial cell apoptosis induced by methamphetamine. Cell Death Dis 7, e2161, doi:10.1038/cddis.2016.67 (2016).

28 Ohoka, N., Yoshii, S., Hattori, T., Onozaki, K. \& Hayashi, H. TRB3, a novel ER stress-inducible gene, is induced via ATF4-CHOP pathway and is involved in cell death. EMBO J 24, 1243-1255, doi:10.1038/sj.emboj.7600596 (2005).

29 Parone, P. A. et al. Preventing mitochondrial fission impairs mitochondrial function and leads to loss of mitochondrial DNA. PLoS One 3, e3257, doi:10.1371/journal.pone.0003257 (2008).

30 Gottlieb, E., Armour, S. M., Harris, M. H. \& Thompson, C. B. Mitochondrial membrane potential regulates matrix configuration and cytochrome c release during apoptosis. Cell Death Differ 10, 709-717, doi:10.1038/sj.cdd.4401231 (2003).

31 Chaudhari, N., Talwar, P., Parimisetty, A., Lefebvre d'Hellencourt, C. \& Ravanan, P. A molecular web: endoplasmic reticulum stress, inflammation, and oxidative stress. Front Cell Neurosci 8, 213, doi:10.3389/fncel.2014.00213 (2014).

32 Goruppi, S. \& lovanna, J. L. Stress-inducible protein p8 is involved in several physiological and pathological processes. J Biol Chem 285, 1577-1581, doi:10.1074/jbc.R109.080887 (2010).

33 Rao, J. et al. C/EBP homologous protein (CHOP) contributes to hepatocyte death via the promotion of ERO1alpha signalling in acute liver failure. Biochem $J \mathbf{4 6 6}, 369-378$, doi:10.1042/BJ20140412 (2015).

34 Cahill, T. J. et al. Resistance of Dynamin-related Protein 1 Oligomers to Disassembly Impairs Mitophagy, Resulting in Myocardial Inflammation and Heart Failure. J Biol Chem 290, 25907-25919, doi:10.1074/jbc.M115.665695 (2015).

35 Twig, G. et al. Fission and selective fusion govern mitochondrial segregation and elimination by autophagy. EMBO J 27, 433-446, doi:10.1038/sj.emboj.7601963 (2008).

36 Shirakabe, A. et al. Drp1-Dependent Mitochondrial Autophagy Plays a Protective Role Against Pressure Overload-Induced Mitochondrial Dysfunction and Heart Failure. Circulation 133, 1249-1263, doi:10.1161/CIRCULATIONAHA.115.020502 (2016). 

mammalian heart and brain. EMBO J 33, 2798-2813, doi:10.15252/embj.201488658 (2014).

\section{Figures}
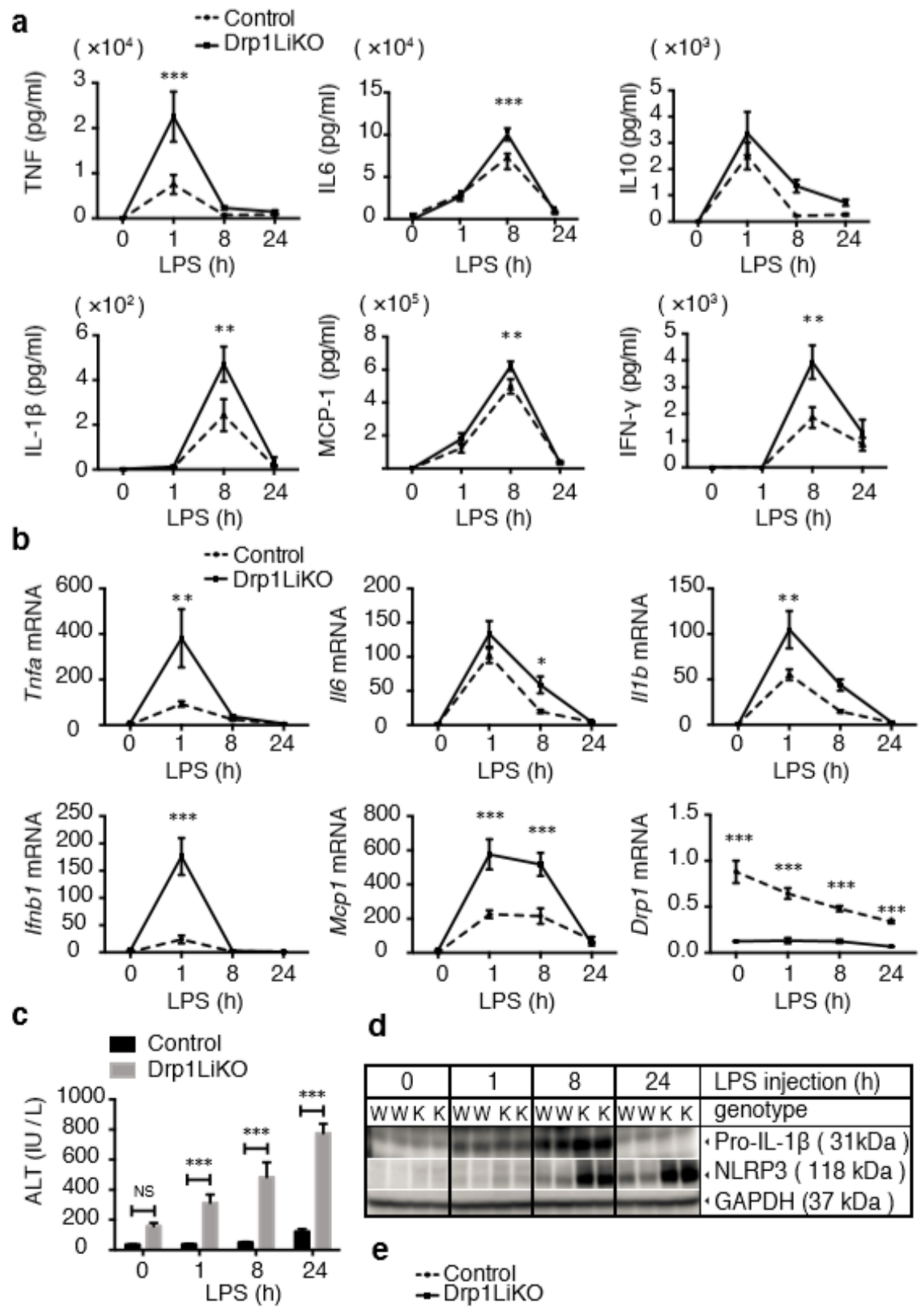

d

LPS (h)
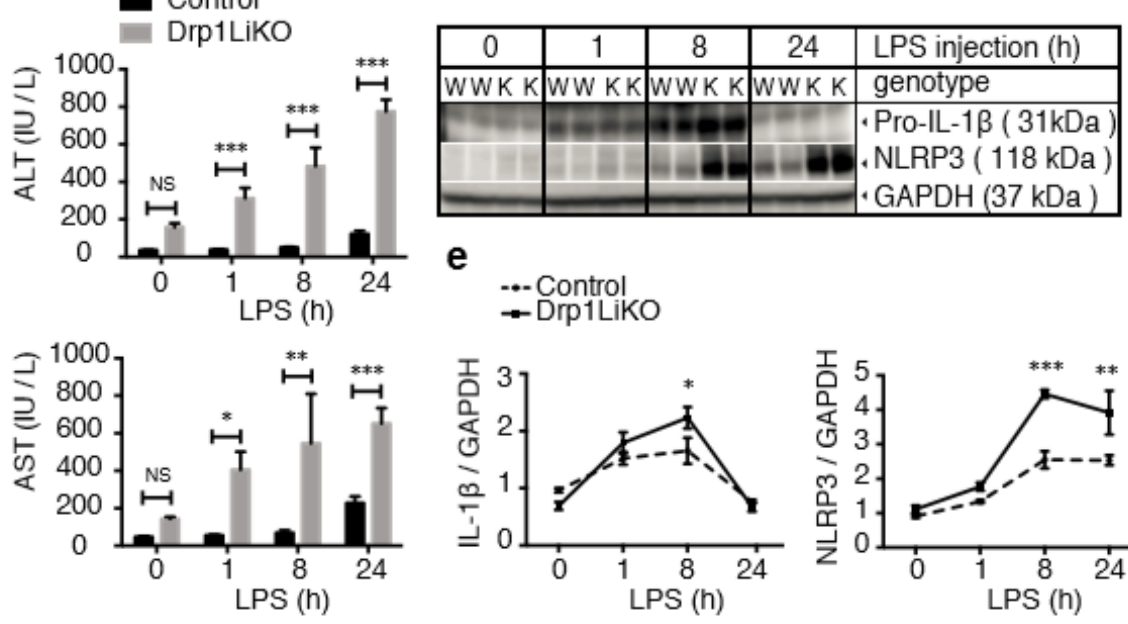

-.-Dontrol
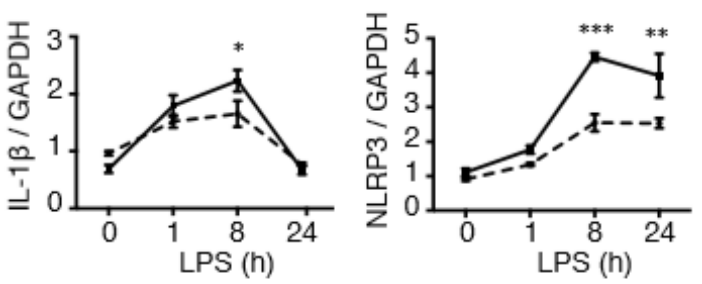

Figure 1 
LPS-induced inflammatory response was augmented in Drp1LiKO mice. Control and Drp1LiKO mice were treated with saline alone (referred to as LPS OH) or LPS ( $5 \mathrm{mg} / \mathrm{kg}$, IP). (a) At indicated time points after LPS treatment, serum samples were collected. Serum TNF, IL-6, IL-10, IL-1 $\beta$, MCP1, and IFN-y levels were determined by BD CBA. $n=5-7$ (b) Tnfa, Il6, II1b, Ifnb1, Mcp1, and Drp1 expression was determined by quantitative real-time PCR of total RNA from saline- or LPS-treated mouse liver tissue at indicated time points. Results were normalized to Gapdh expression and are shown as fold-changes relative to gene expression in saline-treated control mice. $n=3-4$ (c) Serum ALT and AST levels in LPS-treated control and Drp1LiKO mice. $n=4-7$ ( $d$ and e) Western blot analysis and densitometric quantification of Pro-IL-1 $\beta$ and NLRP3 expression in saline- or LPS-treated liver lysates. GAPDH served as an internal control. $n=4$ Values are expressed as mean \pm SEM. NS, no significant difference; ${ }^{\star} p<0.05,{ }^{* *} p<0.01,{ }^{* \star *} p<0.001$. LPS, lipopolysaccharide; Drp1LiKO, liver-specific Drp1-knockout; IP, intraperitoneally; TNF, tumor necrosis factor; IL, interleukin; MCP1, monocyte chemoattractant protein-1; IFN-y, interferon gamma; CBA, cytometric bead array; PCR, polymerase chain reaction; Gapdh, glyceraldehyde 3-phosphate dehydrogenase; ALT, alanine aminotransferase; AST, aspartate transaminase; NLRP3, NLR family pyrin domain containing 3; SEM, standard error of the mean.

a

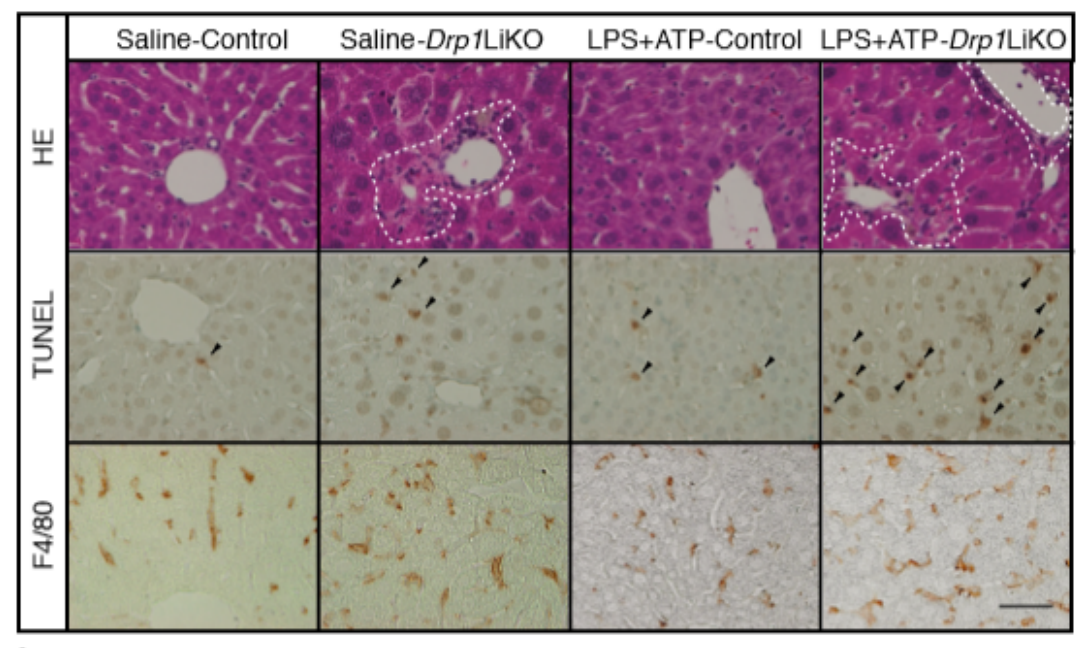

b $\square$ Control Drp1LiKO

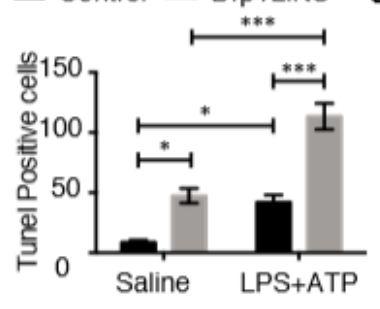

d



Figure 2

LPS/ATP-induced hepatocyte apoptosis and macrophage activation in control and Drp1LiKO mice. Control and Drp1LiKO mice were treated with LPS (10 mg/kg body weight, IP) for $4 \mathrm{~h}$, followed by an additional $50 \mu \mathrm{L}$ IP injection of 100 mM ATP 30 min before euthanization. (a) H\&E staining, TUNEL assay, and F4/80 immunostaining were performed on saline- or LPS/ATP-treated liver sections to examine histology. Areas with inflammatory cell infiltration are outlined by white dashed lines, and TUNEL-positive 
cells are indicated by arrowheads. Scale bar $=50 \mu \mathrm{m}$. (b) Quantification of TUNEL-positive cells in salineor LPS/ATP-treated control and Drp1LiKO mice. Values are expressed as mean $\pm S E M . n=3 .{ }^{*} p<0.05$, ${ }^{* *} p<0.01,{ }^{* \star} p<0.001$. (c) Quantitative analysis of the number of $F 4 / 80$-positive cells performed by counting cells in 15 high-power fields (20x) per slide from 4 mice per group. Values are expressed as mean \pm SEM. $n=4$. NS, no significant difference; ${ }^{\star} p<0.05,{ }^{*} p<0.01,{ }^{\star \star *} p<0.001$. (d) Representative images of IL-1 $\beta$ and F4/80 staining of liver tissue from LPS-/ATP-treated mice. IL-1 $\beta$-positive cells were visualized using Alexa Fluor 594-conjugated chicken anti-goat IgG (red) and F4/80-positive cells using Alexa Fluor 488-conjugated rabbit anti-rat IgG (green). Nuclei were stained with DAPI (blue). Localization of Kupffer cells in the hepatic sinusoid is indicated (white dashed lines). Areas indicated with white dashed squares are enlarged and shown on the right side of the picture. Scale bar $=10 \mu \mathrm{m}$. LPS, lipopolysaccharide; ATP, adenosine triphosphate; Drp1LiKO, liver-specific Drp1-knockout; H\&E, hematoxylin and eosin; TUNEL, terminal deoxynucleotidyl transferase dUTP nick end labeling; IP, intraperitoneally; SEM, standard error of the mean; IgG, immunoglobulin G; DAPI, 4',6-diamidino-2phenylindole. 



b M2 Macrophage ( $7 \mathrm{AAD}^{-} \mathrm{CD} 45^{+} \mathrm{CD} 11 \mathrm{~b}^{+} \mathrm{F} 4 / 80^{+} \mathrm{CD} 206^{\mathrm{High}}$ )
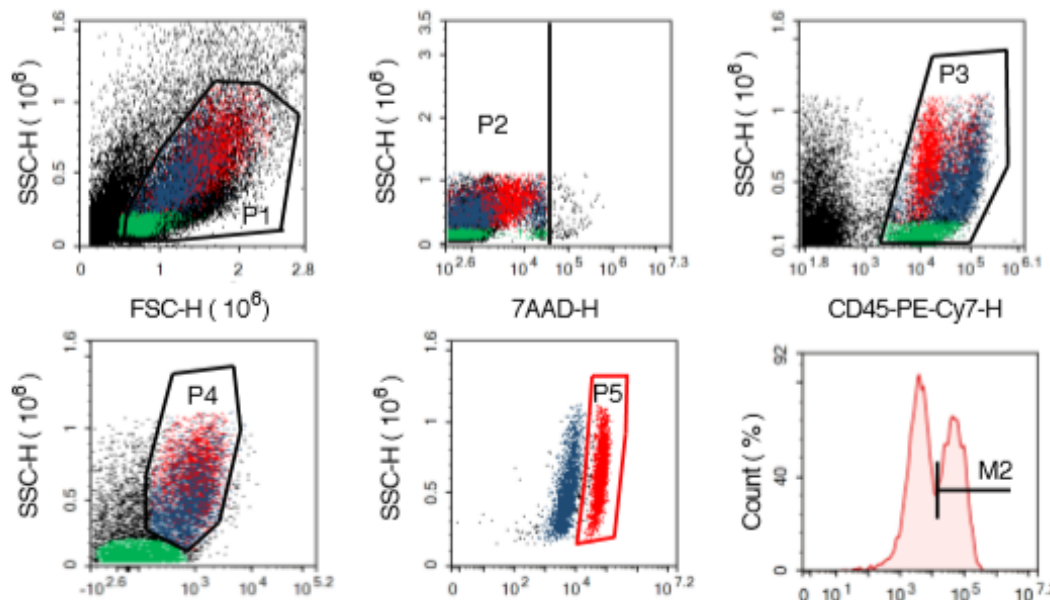

CD11b-APC-Cy7-H



CD45-PE-Cy7-H
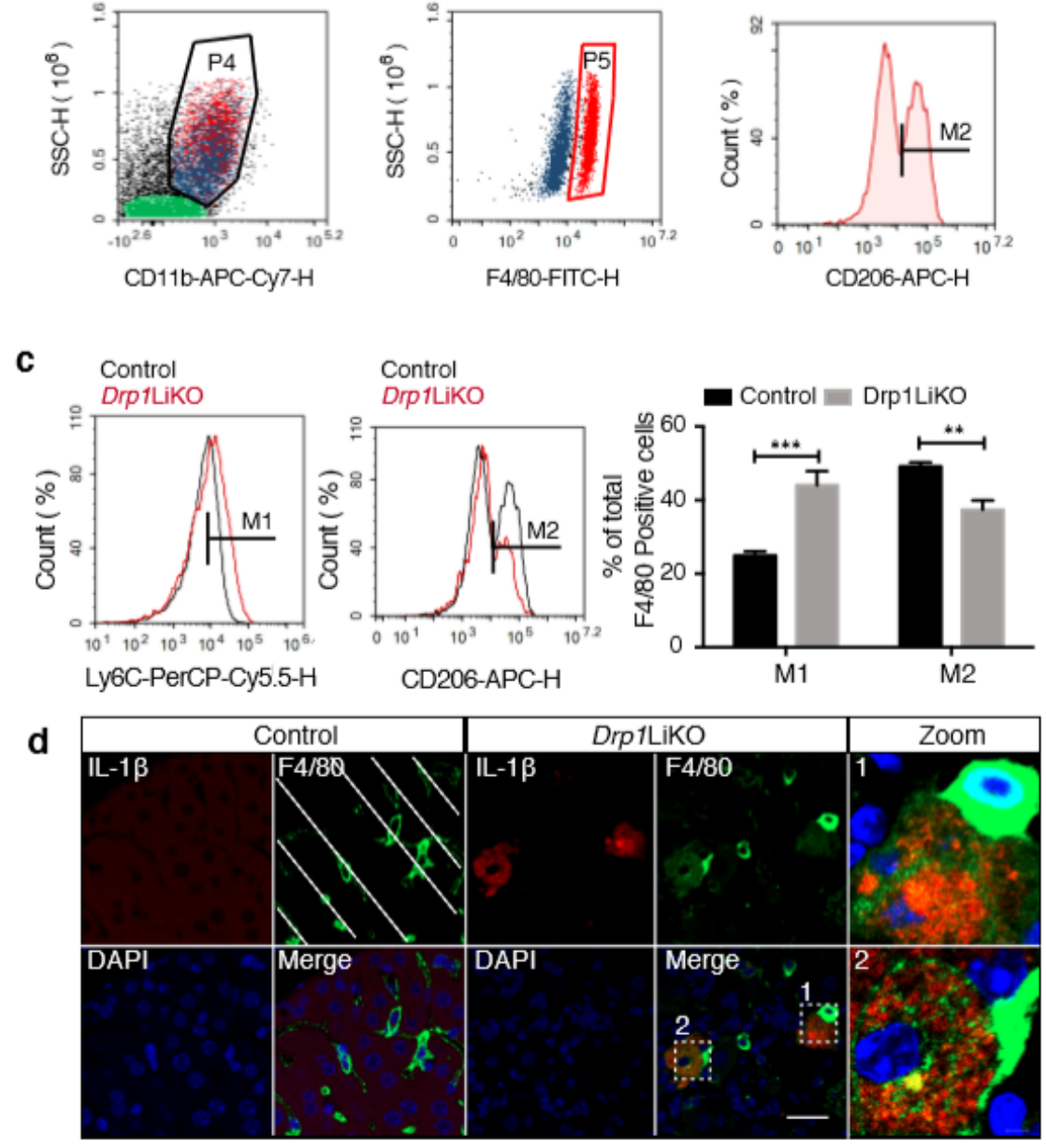

\section{Figure 3}

DRP1 ablation increases inflammatory macrophage infiltration in Drp1LiKO mice. (a and b) Macrophage gating strategy and percentage analysis. Gating strategy and percentage analysis of M1 (CD45+CD11b+ F4/80+Ly6CHigh) and M2 (7AAD-CD45+CD11b+ F4/80+CD206High) macrophages. Cell populations were gated sequentially from left to right. (c) Percentage analysis of M1 (Ly6CHigh) and M2 (CD206High) macrophages isolated from liver tissue of NCD mice. Values are expressed as mean \pm SEM. $n=4$. ${ }^{*} p<$ 
$0.01, * \star \star p<0.001$. (d) Representative images of IL-1 $\beta$ and F4/80 staining of liver tissue of NCD mice. IL$1 \beta$-positive cells were visualized using Alexa Fluor 594-conjugated chicken anti-goat IgG (red) and F4/80-positive cells using Alexa Fluor 488-conjugated rabbit anti-rat IgG (green). Nuclei were stained with DAPI (blue). Localization of Kupffer cells in the hepatic sinusoid is indicated (white dashed lines). Areas indicated with white dashed squares are enlarged and shown on the right side of the picture. Scale bar $=50 \mu \mathrm{m}$. Drp1LiKO, liver-specific Drp1-knockout; NCD, normal chow diet; SEM, standard error of the mean; IL, interleukin; IgG, immunoglobulin G; DAPI, 4',6-diamidino-2-phenylindole.



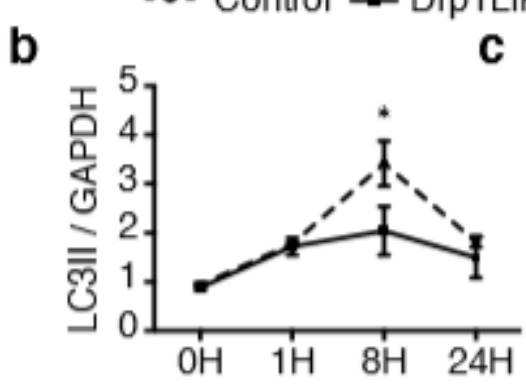

C

d

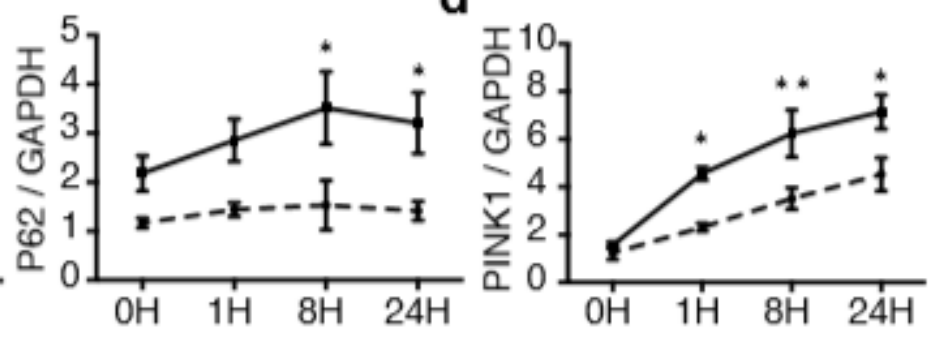

e






\section{Figure 4}

DRP1 defects decrease autophagy in Drp1LiKO mice. (a-d) Control and Drp1LiKO mice were treated with saline alone (referred to as LPS OH) or LPS $(5 \mathrm{mg} / \mathrm{kg}, \mathrm{IP})$. At indicated time points after LPS treatment, liver tissue was collected. Liver LC3, P62, and PINK1 levels were determined by western blot analysis. (bd) Densitometric quantification of LC3, P62, and PINK1 expression in saline- or LPS-treated liver lysates. GAPDH served as an internal control. Values are expressed as mean \pm SEM. $n=4 .{ }^{*} p<0.05,{ }^{\star \star} p<0.01$. (e) Representative images of IL-1 $\beta$ and LC3 staining of liver tissue from LPS/ATP-treated mice. IL-1 $\beta$ positive cells were visualized using Alexa Fluor 594-conjugated chicken anti-goat lgG (red) and LC3 positive cells using Alexa Fluor 488-conjugated donkey anti-rabbit IgG (green). Nuclei were stained with DAPI (blue). Areas indicated with white dashed squares are enlarged and shown on the right side of the picture. Arrowheads indicate LC3-positive dots in hepatocytes. Scale bar $=10 \mu \mathrm{m}$. (f) Western blot analysis of LC3 and GAPDH expression in saline- or LPS/ATP-treated liver lysates. The bar graph shows the densitometric quantification of LC3-II. GAPDH served as an internal control. Values are expressed as mean SEM. $n=4$. NS, no significant difference; ${ }^{*} p<0.05$, ${ }^{\star *} p<0.01$. Drp1LiKO, liver-specific Drp1knockout; LPS, lipopolysaccharide; Sqstm1, sequestosome-1; PINK1, phosphatase and tensin homologinduced putative kinase 1; GAPDH, glyceraldehyde 3-phosphate dehydrogenase; IL, interleukin; LC3, light chain 3; ATP, adenosine triphosphate; IgG, immunoglobulin G; SEM, standard error of the mean; DAPI, 4',6diamidino-2-phenylindole. 
a

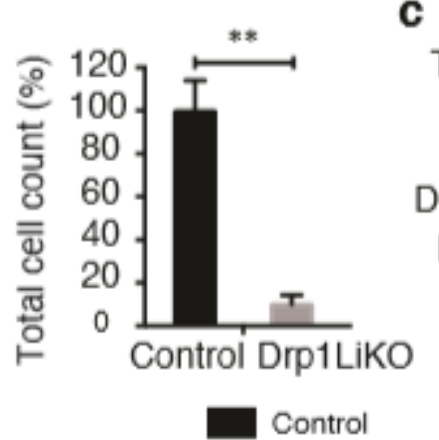

b

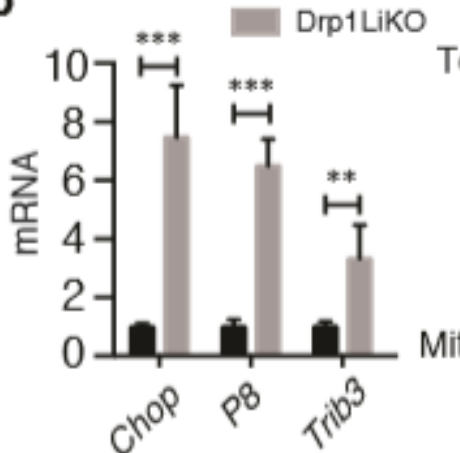

d

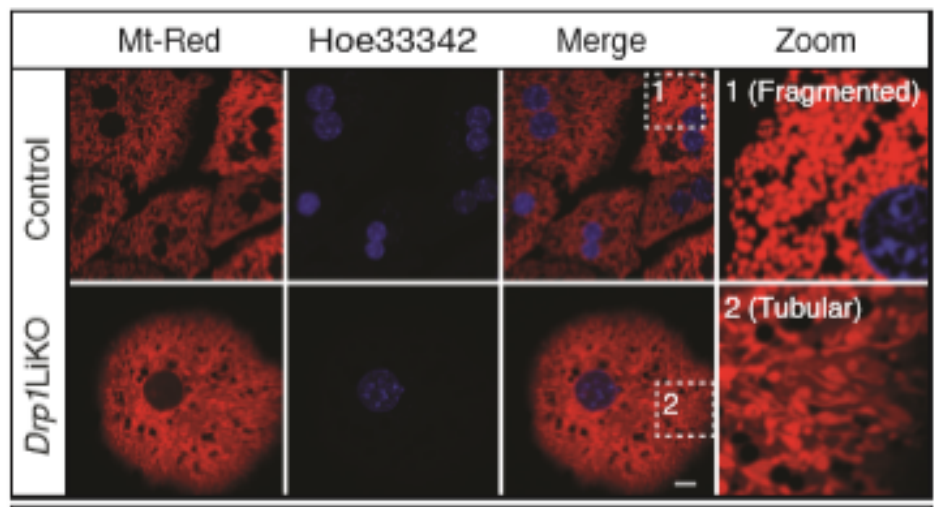

e

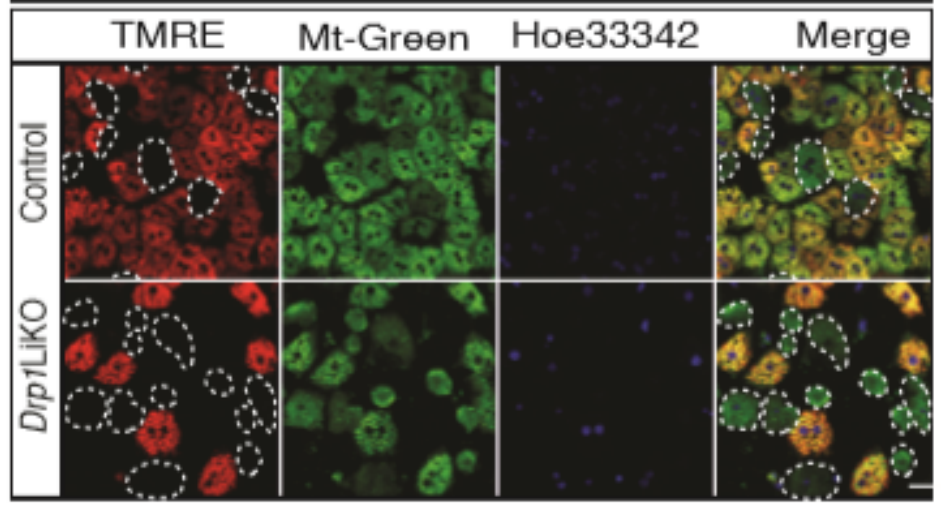

Top 5 enrichment in biological process (up-regulated genes)

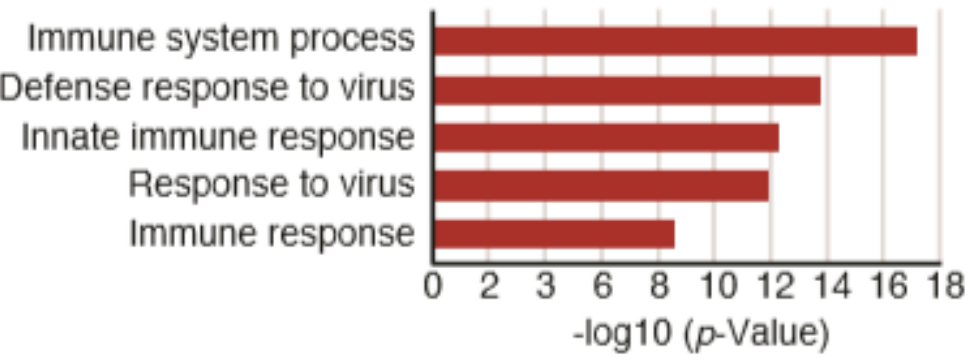

Top 5 enrichment in biological process (down-regulated genes)

Chromosome segregation

Cell cycle Cell division

Chromosome segregation Mitotic chromosome condensation

$\begin{array}{lllll}4 & 7 & 10 & 13 & 16 \\ & -\log 10 & (p-\text { Value })\end{array}$
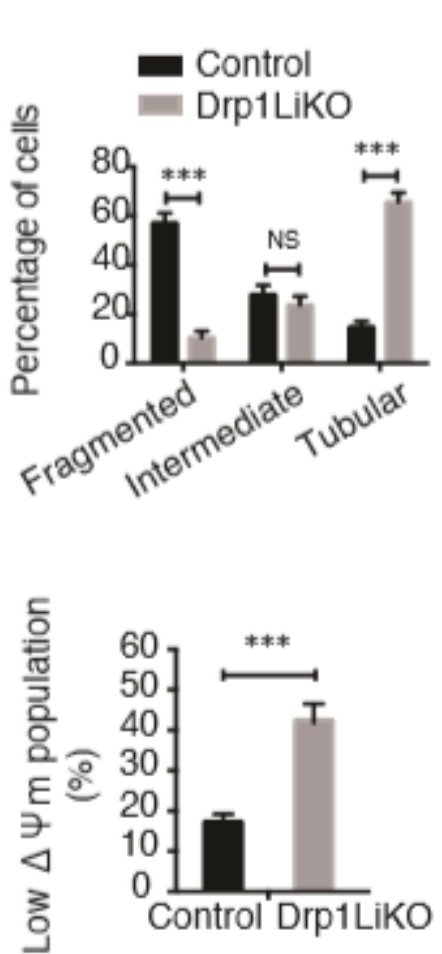

\section{Figure 5}

Microarray functional annotation analysis, mitochondrial morphology, and mitochondrial membrane potential in control and Drp1LiKO mouse primary hepatocytes. (a) Total mouse primary hepatocyte numbers from control and Drp1LiKO mice were measured by trypan blue staining to measure viable cells. Total cell numbers from control mice represent 100\%, and total cell numbers from Drp1LiKO mice were normalized as a percentage of control values. $n=4$. (b) Chop, P8, and Trib3 expression was determined 
by quantitative real-time PCR of total RNA from control and Drp1LiKO mice primary hepatocytes. Results were normalized to Gapdh expression and are shown as fold-changes relative to control mice. Values are expressed as mean \pm SEM. $n=4$. ${ }^{* \star} p<0.01$, ${ }^{\star \star \star} p<0.001 . n=4$. (c) Top five biological process $G 0$ terms of up- and downregulated genes ranked by $p$-value. (d) To visualize mitochondria, control and Drp1LiKO mouse primary hepatocytes were stained with MitoTracker® Red. Nuclei were stained with Hoechst33342, and images were captured with a confocal microscope. Areas indicated with white dashed squares are enlarged and shown on the right. Mitochondrial morphology was graded as fragmented, intermediate, or tubular for control and Drp1LiKO mouse primary hepatocytes. Quantification was performed by counting cells in 3-5 high-power fields (60x) per slide from 3 independent experiments. Scale bar $=10 \mu \mathrm{m}$. (e) Mitochondrial membrane potential $(\Delta \Psi \mathrm{m})$ measured in control and Drp1LiKO mouse primary hepatocytes using the fluorescence probe TMRE. Mitochondria and nuclei were stained by MitoTracker® Green and Hoechst33342, respectively. White dashed lines track cell boundaries with dissipated $\Delta \Psi \mathrm{m}$ (low $\Delta \Psi \mathrm{m}$ ). Quantification of low- $\Delta \Psi_{\mathrm{m}}$ cells was performed by counting cells in three high-power fields $(20 x)$ per slide from three independent experiments. Scale bar $=50 \mu \mathrm{m}$. Drp1 LiKO, liverspecific Drp1-knockout; Chop, C/EBP homologous protein; Trib3, Tribbles homolog 3; PCR, polymerase chain reaction; Gapdh, glyceraldehyde 3-phosphate dehydrogenase; SEM, standard error of the mean; GO, Gene Ontology; TMRE, tetramethylrhodamine ethyl ester perchlorate. 


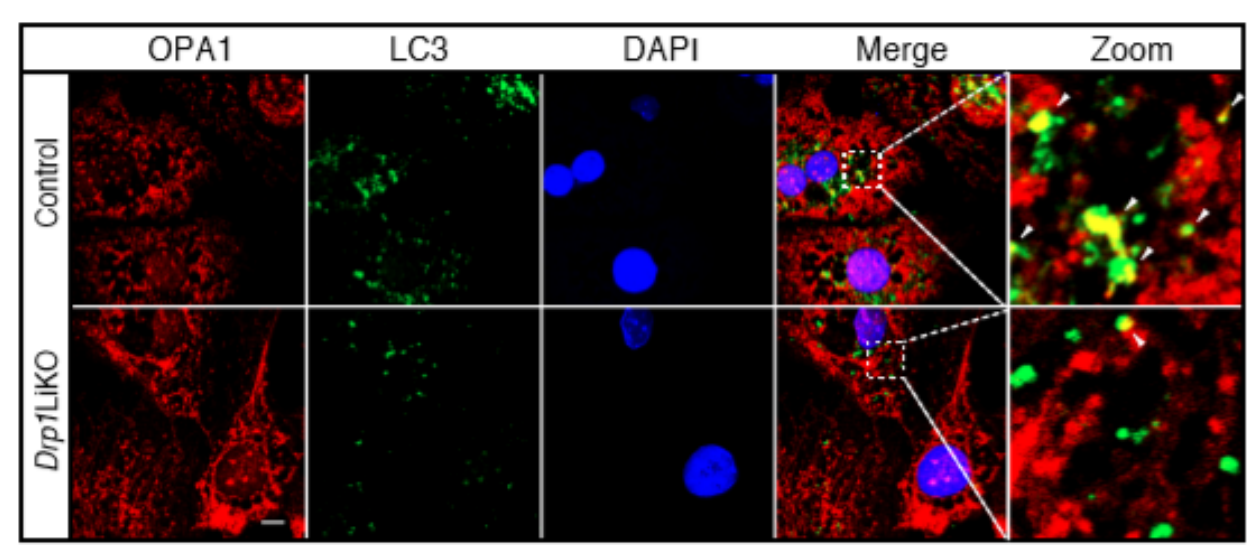

b

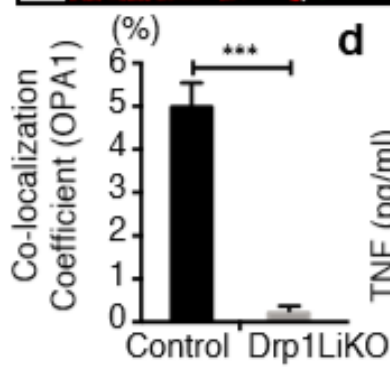

d $\quad \cdots$ Control
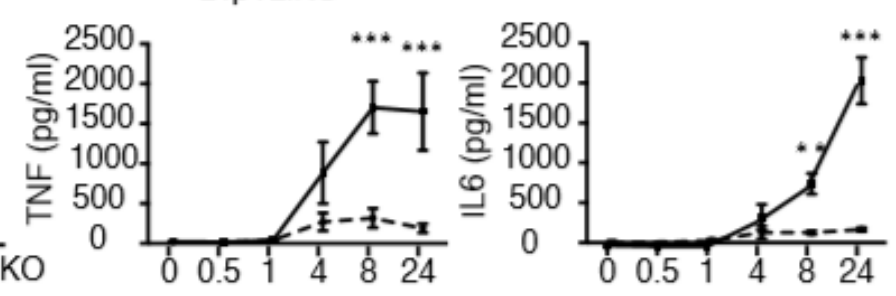

C

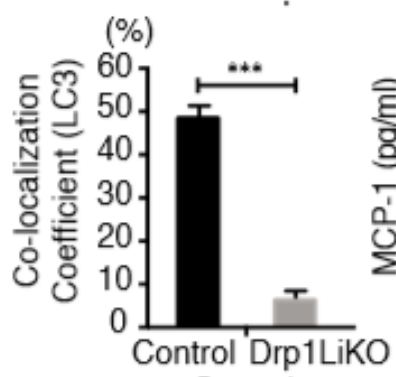

LPS (h)

LPS (h)
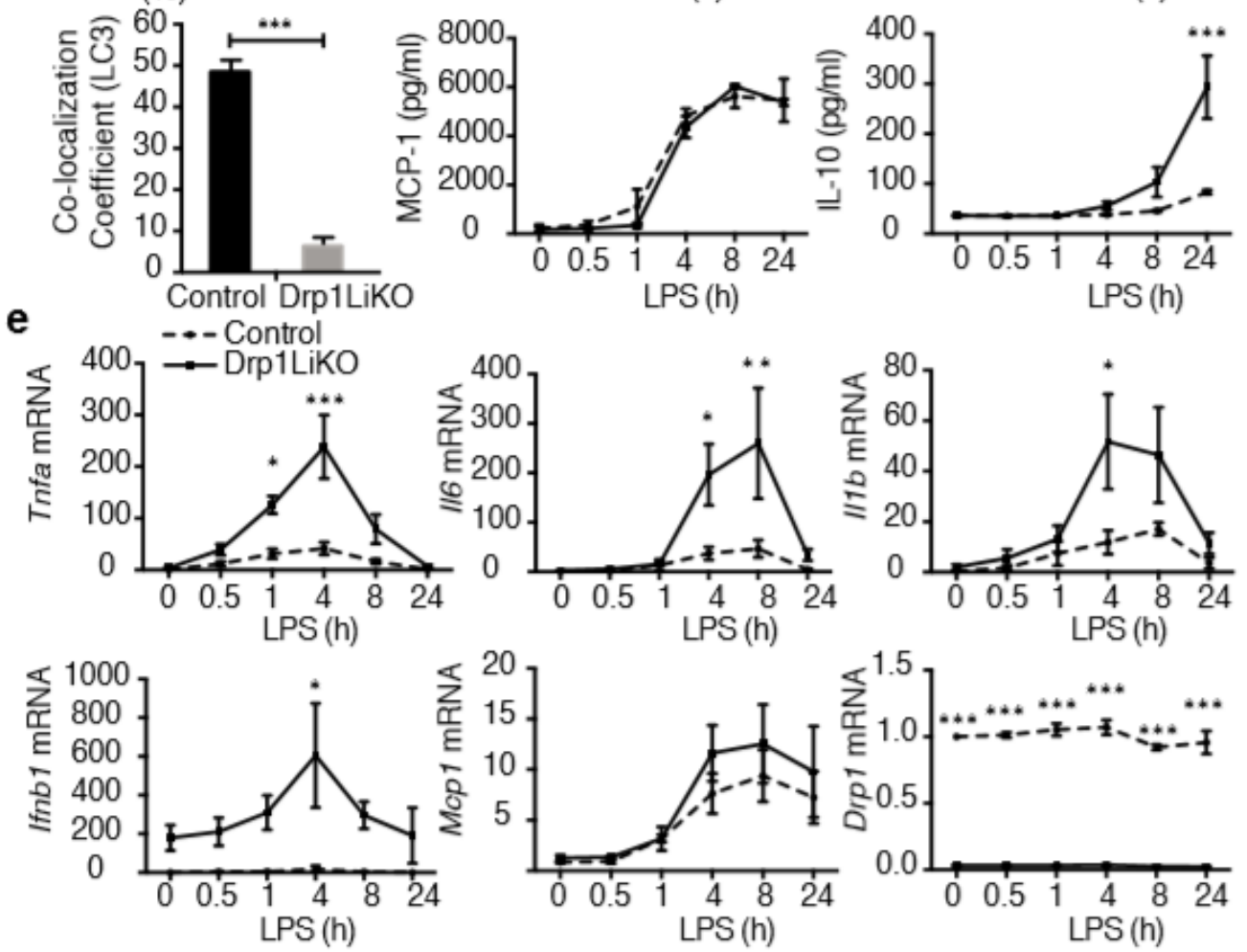

\section{Figure 6}

Decreased mitophagy impairment and increased inflammatory response in Drp1LiKO mouse primary hepatocytes. (a) Representative images of OPA1 and LC3 staining of LPS-treated control and Drp1LiKO mouse primary hepatocytes. OPA1-positive cells were visualized using Alexa Fluor 594-conjugated goat anti-mouse IgG (red) and LC3-positive cells using Alexa Fluor 488-conjugated donkey anti-rabbit IgG (green). Nuclei were stained with DAPI (blue). Areas indicated with white dashed squares are enlarged 
and shown on the right side of the picture. Scale bar $=10 \mu \mathrm{m}$. (b and c) Statistical analysis of the colocalization coefficient of OPA1 and LC3. Control $n=3$ experiments, Drp1LiKO $n=5$ experiments. (b) The co-localization coefficient was represented as a percentage of LC3-positive OPA1 punctate signals. (c) The co-localization coefficient was represented as a percentage of OPA1-positive LC3 punctate signals.

(d) At indicated time points after LPS treatment, culture supernatants were collected. TNF, IL-6, MCP1, and IL-10 levels were determined by BD CBA. $n=3-5 . * * p<0.01$, $* \star * p<0.001$. (e) Total RNA was isolated at indicated time points after LPS treatment, and Tnfa, II6, II1b, Ifnb1, Mcp1, and Drp1 expression was determined by quantitative real-time PCR. Results were normalized to Gapdh expression and are shown as fold-changes relative to gene expression in untreated control cells. $n=3$. Values are expressed as mean \pm SEM. ${ }^{*} p<0.05,{ }^{* \star} p<0.01,{ }^{* \star} p<0.001$. Drp1LiKO, liver-specific Drp1-knockout; OPA1, optic atrophy 1; LC3, light chain 3; IgG, immunoglobulin G; DAPI, 4',6-diamidino-2-phenylindole; LPS, lipopolysaccharide; IL, interleukin; TNF, tumor necrosis factor; IL, interleukin; MCP1, monocyte chemoattractant protein-1; IFN-ץ, interferon gamma; CBA, cytometric bead array; Gapdh, glyceraldehyde 3phosphate dehydrogenase; SEM, standard error of the mean.

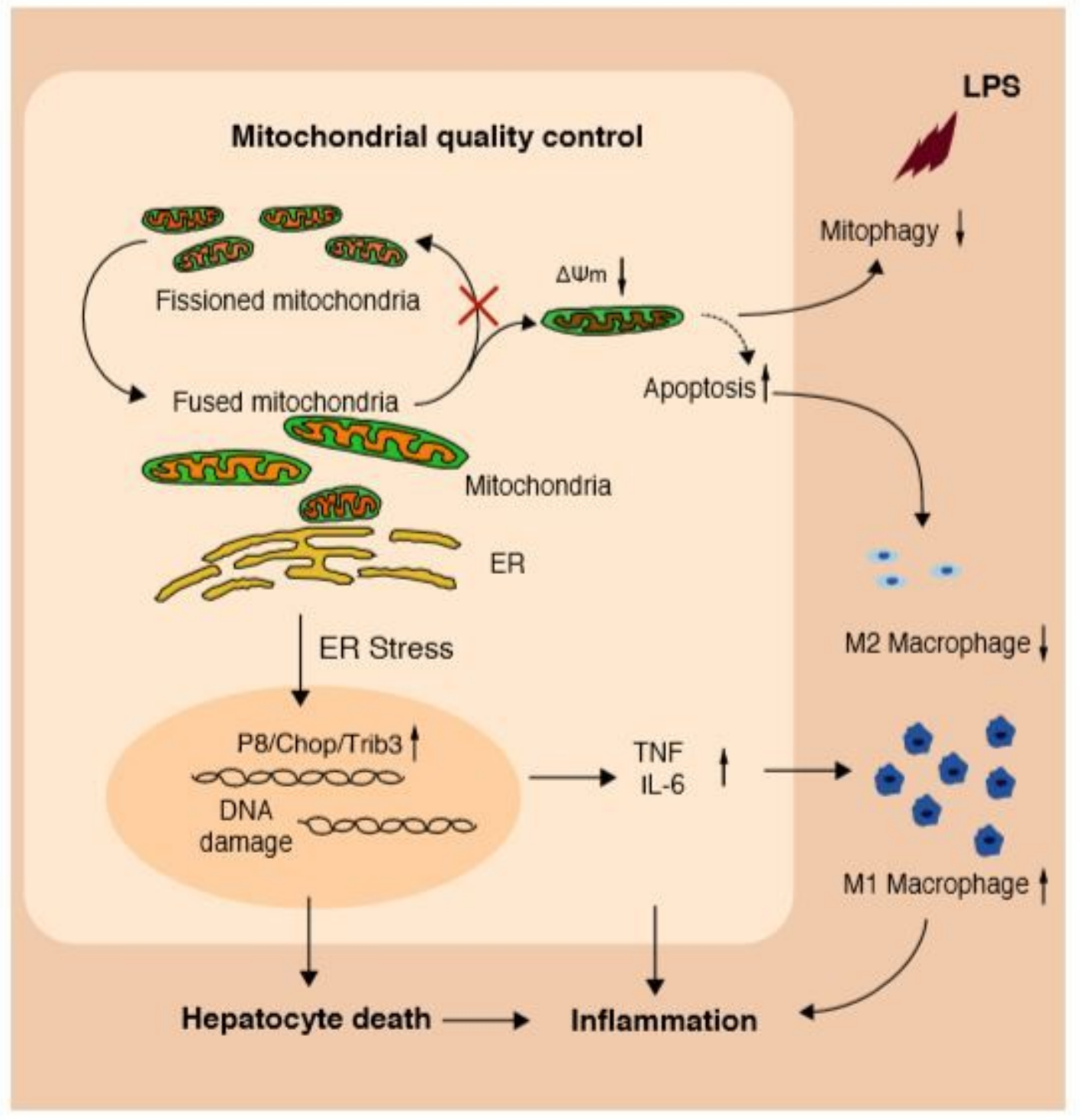


Schematic model of macrophage activation and liver inflammation. Communication defects between mitochondria and the ER upregulate transcription factors P8, Chop, and Trib3, which, in turn, increase hepatocyte apoptosis. Other possible mechanisms for hepatocyte apoptosis is low $\Delta \Psi \mathrm{m}$ and increased DNA damage induced by mitochondrial dynamics defects. These dead cells, in turn, stimulate macrophage activation and polarization. M1-polarized inflammatory macrophages were significantly increased because of hepatocyte apoptosis, in turn accelerating the inflammatory response after an LPS challenge. Mitophagy defects also contribute to the elevated inflammatory response in Drp1LiKO mice. Chop, C/EBP homologous protein; Trib3, Tribbles homolog 3; ER, endoplasmic reticulum; Drp1LiKO, liverspecific Drp1-knockout; LPS, lipopolysaccharide.

\section{Supplementary Files}

This is a list of supplementary files associated with this preprint. Click to download.

- Fig17SupFig14Tab15.pdf 